\title{
Decision Trees And Quality Control Decisions
}

Awni Zebda (E-mail: azebda@cob.tamucc.edu), Texas A\&M University-Corpus Christi

\begin{abstract}
Bayesian decision tree analysis has been widely used as a basis for quality control decision making. Recently, the traditional decision tree analysis has been criticized for requiring a lot of calculations and, therefore, being inefficient. This paper presents a simplified and efficient decision tree analysis for quality control decision making that improves the efficiency of the traditional decision analysis by reducing substantially the number of calculations required to solve decision problems. For some decision problems, the proposed analysis reduces the number of calculations required to solve decision problems by more than $75 \%$.
\end{abstract}

Some researchers provided modified decision trees (Game trees and Scenario trees) that attempt to preserve the advantages of the traditional trees while improving their efficiency. However, these other modified decision trees may not be as efficient as the traditional analysis because they do not allow for the use of the coalescence procedure in the case of symmetrical decision problems.

\section{Introduction}

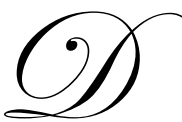

ecision trees are widely used as a basis for quality control decision making. Recently, the traditional decision tree analysis has been criticized for requiring a lot of calculations and, therefore, being inefficient. This paper presents a modified decision tree analysis for quality control decision making that improves the efficiency of the traditional decision analysis by reducing substantially the number of calculations required to solve decision problems. As Shown in the paper, the modified analysis may save more than $75 \%$ of the operations required by the traditional decision analysis.

Other researchers have provided modified decision trees, including Game trees [Shenoy, 1993b] and Scenario trees [Shenoy, 1994b], to address the problem of the inefficiency of the traditional decision trees. These modified decision trees, like the modified decision tree analysis proposed in this paper, attempt to preserve the advantages of the traditional trees while improving the efficiency of the solution. However, as shown in the paper, these modified decision trees may not be as efficient as the traditional tree analysis for some decision problems.

The paper is divided into seven sections. The next section discusses the traditional decision tree. Section three presents the modified decision tree analysis. Section four uses an example of a single-level decision tree to compare the modified and traditional decision tree analysis. Section five discusses mathematically the advantages of the modified analysis in the cases of single-level and multi-level trees. Section six compares the proposed modified decision tree analysis to scenario and game trees suggested by other researchers as modifications to the traditional analysis. Finally, section seven provides a summary and concluding remarks.

\section{The Traditional Decision Tree Analysis}

Bayesian decision tree analysis has its foundations in decision theory [van Neumann and Morgenstern, 1944]. It graphically depicts all possible combinations of decisions and states of nature (random events). Decision variables are represented by rectangles (called decision nodes) and random variables are represented by circles (called chance nodes). Arrows pointing into a decision variable (decision node) show the information available at 
the time the decision is made and arrows pointing to a random variable (chance node) show the existence of a conditional probability distribution for the random variable.

The solution procedure of decision trees allows for the computation of an optimal decision procedure by using the traditional rollback method or the "averaging out" and "folding back" procedure [Raiffa, 1968]. More specifically, joint and marginal probabilities are obtained and used with Bayes Theorem to obtain the conditional (revised) probabilities. Then, the optimal decision procedure and its expected value are obtained by the rollback method. That is, starting from the leaves (endpoints of the tree), random and decisions variables (nodes) are deleted recursively. Random nodes are deleted by the averaging out procedure (taking the average of the payoffs at the end of their edges with the probabilities at the nodes being used as weights). The decision nodes are deleted by the folding back procedure (maximizing the payoffs at the end of their edges).

Mathematically, let the prior probability of state of nature $\left(\mathrm{s}_{\mathbf{i}}\right)$ be denoted by $\mathrm{P}\left(\mathrm{s}_{\mathrm{i}}\right)$ and the probability of a signal $\mathrm{y}_{\mathrm{k}}$ given $\mathrm{s}_{\mathrm{i}}$ be denoted by $\mathrm{P}\left(\mathrm{y}_{\mathrm{k}} / \mathrm{s}_{\mathrm{i}}\right)$. Then, the joint, marginal, and conditional probabilities are given, respectively by:

$\mathrm{P}\left(\mathrm{s}_{\mathrm{i}}, \mathrm{y}_{\mathrm{k}}\right)=\mathrm{P}\left(\mathrm{s}_{\mathrm{i}}\right) * \mathrm{P}\left(\mathrm{y}_{\mathrm{k}} / \mathrm{s}_{\mathrm{i}}\right)$

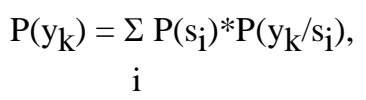

$\mathrm{P}\left(\mathrm{s}_{\mathrm{i}} / \mathrm{y}_{\mathrm{k}}\right)=\mathrm{P}\left(\mathrm{y}_{\mathrm{k}}, \mathrm{s}_{\mathrm{i}}\right) / \mathrm{P}\left(\mathrm{y}_{\mathrm{k}}\right)$.

The expected value of the optimal procedure is calculated by:

$\mathrm{EV}=\underset{\mathrm{k}}{\Sigma}\left[\max \Sigma \mathrm{w}\left(\mathrm{s}_{\mathrm{i}}, \mathrm{d}_{\mathrm{j}}\right) * \mathrm{P}\left(\mathrm{s}_{\mathrm{i}} / \mathrm{y}_{\mathrm{k}}\right)\right] * \mathrm{P}\left(\mathrm{y}_{\mathrm{k}}\right)$
$\quad$

where $\mathrm{w}\left(\mathrm{s}_{\mathrm{i}}, \mathrm{d}_{\mathrm{j}}\right)$ is the payoff associated with state $\mathrm{s}_{\mathrm{i}}$ and action $\mathrm{d}_{\mathrm{j}}$ and the conditional probability $\mathrm{P}\left(\mathrm{s}_{\mathrm{i}} / \mathrm{y}_{\mathrm{k}}\right)$ is obtained by Equation (3).

\section{A Modified Decision Tree Analysis}

Decision trees can be classified as symmetrical (if each path from the root node to a leaf node includes the same random variables and decision variable) or non-symmetrical. Decision trees can also be classified as one-level or multi-level. A decision tree is considered to be one-level when only one level of signals is assumed. Multi-level decision trees involve multiple levels of signals and are very common in many real life decisions. For example, a quality control engineer may conduct a more detailed test after conducting a less detailed test to decide whether to accept a product or process as safe. In this case, the tree will have two levels instead of one level.

The traditional decision tree analysis can be used in the cases of single-level and multi-level trees. It can also be applied to symmetrical and non-symmetrical problems. In the case of symmetrical trees, the traditional tree analysis allows for the use of the coalescence procedure [Olmsted, 1983] which reduces the number of operations needed to obtain the solution without affecting the number of operations needed to reprocess probabilities.

The traditional decision tree analysis can be criticized for requiring a lot of calculations and, thus, being inefficient. Consider, for example, a problem with two decisions $(\mathrm{m}=2)$, ten states of nature $(\mathrm{n}=10)$, and one-level signal. In this case, as explained later, the traditional tree analysis requires 699 operations to obtain the solution (290 to reprocess the probabilities and 409 to obtain the optimal decision). If the decision problem has two-levels signals, the number of operations needed to solve the problem will be 7399 (3190 to reprocess probabilities and 4209 to obtain the optimal decision). 
The inefficiency of the traditional decision tree analysis has been noted by many management scientists (Kirkwood [1993], Quinlan [1987], Shachter [1986, 1988], Smith [1989], Shenoy [1989, 1992]). However, the traditional decision analysis can be simplified and the number of calculations can be substantially reduced. In fact, some researchers have proposed modified decision trees, including Game trees [Shenoy, 1993b] and Scenario trees [Shenoy, 1994b], that attempt to preserve the advantages of the traditional analysis while improving the efficiency of the solution. However, these modified decision trees may not be as efficient as the traditional trees because, as explained later, they do not allow for using the coalescence procedure when the decision problem is symmetrical.

The remainder of this section presents a modified decision tree analysis that substantially reduces the number of calculations by eliminating the need for calculating the revised (conditional) probabilities. The simplified analysis can be derived as follows. Substituting (3) into (4) result in:

$$
\begin{gathered}
\left.\mathrm{EV}=\underset{\mathrm{k}}{\Sigma[\max } \Sigma \mathrm{i}\left(\mathrm{s}_{\mathrm{i}}, \mathrm{d}_{\mathrm{j}}\right) *\left(\mathrm{p}\left(\mathrm{y}_{\mathrm{k}}, \mathrm{s}_{\mathrm{i}}\right) / \mathrm{p}\left(\mathrm{y}_{\mathrm{k}}\right)\right)\right] * \mathrm{p}\left(\mathrm{y}_{\mathrm{k}}\right) .
\end{gathered}
$$

Equation (5) can be simplified as:

$$
\begin{aligned}
& \mathrm{EV}=\Sigma\left[\max \Sigma \mathrm{w}\left(\mathrm{s}_{\mathbf{i}}, \mathrm{d}_{\mathbf{j}}\right)^{*} \mathrm{p}\left(\mathrm{y}_{\mathbf{k}}, \mathrm{s}_{\mathbf{i}}\right)\right] . \\
& \text { k j i }
\end{aligned}
$$

Then, using (2) in (6) results in the following simplified equation for calculating the expected value:

$$
\begin{aligned}
& \mathrm{EV}=\Sigma\left[\max \Sigma \mathrm{p}\left(\mathrm{s}_{\mathrm{i}}\right)^{*} \mathrm{p}\left(\mathrm{y}_{\mathrm{k}} / \mathrm{s}_{\mathrm{i}}\right)^{*} \mathrm{w}\left(\mathrm{s}_{\mathbf{i}}, \mathrm{d}_{\mathrm{j}}\right)\right], \\
& \text { k j i }
\end{aligned}
$$

where $\mathrm{w}\left(\mathrm{s}_{\mathrm{i}}, \mathrm{d}_{\mathrm{j}}\right), \mathrm{p}\left(\mathrm{s}_{\mathrm{i}}\right)$, and $\mathrm{p}\left(\mathrm{y}_{\mathrm{k}} / \mathrm{s}_{\mathrm{i}}\right)$ are as defined above.

As the above equation suggests, the proposed model requires the calculation of only joint probabilities and eliminates the need to calculate marginal and conditional probabilities. That is, the proposed model reduces the need for the reprocessing of probabilities required by the traditional decision trees.

The proposed analysis can be used in the cases of single-level and multi-level trees. It can also be applied to both symmetrical and non-symmetrical decision problems. In the case of symmetrical problems, like the traditional analysis, the proposed model allows for the use of the coalescence procedure. The savings obtained by the proposed model over the traditional trees are illustrated and discussed in the next two sections.

\section{Numerical Illustration}

To compare the modified and the traditional decision trees, consider the problem of planning quality control tests faced by the quality control department. The quality control engineer is assumed to face two alternative actions. The first is to accept the process as safe $\left(\mathrm{d}_{1}\right)$ and the second is to require an improvement of the process $\left(\mathrm{d}_{2}\right)$. The two states of nature are SAFE PROCESS $\left(\mathrm{s}_{1}\right)$ and UNSAFE PROCESS $\left(\mathrm{s}_{2}\right)$.

Assume that the engineer estimated the prior probabilities of the states of the process to be $\mathrm{P}\left(\mathrm{s}_{1}\right)=.60$ and $\mathrm{P}\left(\mathrm{s}_{2}\right)=.40$. Moreover, assume that the payoffs associated with the different combinations of alternative actions and states of nature are given by the following matrix:

\begin{tabular}{|crr|}
\hline Actions & \multicolumn{2}{c|}{ States of Nature } \\
\cline { 2 - 3 } & $\mathrm{S}_{1}$ & $\mathrm{~S}_{2}$ \\
\hline $\mathrm{d}_{1}$ & 7,000 & $-20,000$ \\
$\mathrm{~d}_{2}$ & $-1,000$ & 3,000 \\
\hline
\end{tabular}


The engineer is considering the use of a testing procedure to collect additional evidence about the state of the process. The procedure provides one of two signals $\left(\mathrm{y}_{1}=\right.$ SAFE PROCESS and $\mathrm{y}_{2}=$ UNSAFE PROCESS). The estimated cost of the procedure is $\$ 500$. The procedure is known to provide correct predictions $80 \%$ of the time and wrong predictions $20 \%$ of the time. That is, the following probabilities are estimated: $\mathrm{P}\left(\mathrm{y}_{1} / \mathrm{s}_{1}\right)$ $=.80, \mathrm{P}\left(\mathrm{y}_{2} / \mathrm{s}_{1}\right)=.20, \mathrm{P}\left(\mathrm{y}_{1} / \mathrm{s}_{2}\right)=.20$, and $\mathrm{P}\left(\mathrm{y}_{2} / \mathrm{s}_{2}\right)=.80$.

Using the traditional analysis, the revised probabilities are obtained in Figure 1.

Figure 1: The Reprocessing Of Probabilities

\begin{tabular}{|c|c|c|c|c|}
\hline & Prior & & Joint & Conditional \\
\hline & $\mathbf{P}\left(\mathbf{s}_{\mathbf{i}}\right)$ & $\mathbf{P}\left(\mathbf{y}_{1} / \mathbf{s}_{\mathbf{i}}\right)$ & $\mathbf{P}\left(\mathbf{y}_{1}, \mathbf{s}_{\mathbf{i}}\right)$ & $\mathbf{P}\left(\mathbf{s}_{\mathbf{i}} / \mathbf{y}_{1}\right)$ \\
\hline $\mathbf{s}_{1}$ & .6 & .8 & .48 & $(.48 / .56)=.857$ \\
\hline $\mathbf{s}_{2}$ & .4 & .2 & .08 & $(.08 / .56)=.143$ \\
\hline & Marginal & $\mathrm{P}\left(\mathrm{y}_{1}\right)$ & .56 & \\
\hline
\end{tabular}

If $\mathbf{y}_{\mathbf{2}}$ :
\begin{tabular}{|ccccc|}
\hline & Prior & Joint & Conditional \\
\cline { 2 - 5 } & $\mathbf{P}\left(\mathbf{s}_{\mathbf{i}}\right)$ & $\mathbf{P}\left(\mathbf{y}_{\mathbf{1}} / \mathbf{s}_{\mathbf{i}}\right)$ & $\mathbf{P}\left(\mathbf{y}_{\mathbf{1}}, \mathbf{s}_{\mathbf{i}}\right)$ & $\mathbf{P}\left(\mathbf{s}_{\mathbf{i}} / \mathbf{y}_{\mathbf{1}}\right)$ \\
\hline $\mathbf{s}_{\mathbf{1}}$ & .6 & .2 & .12 & $(.12 / .44)=.273$ \\
$\mathbf{s}_{\mathbf{2}}$ & .4 & .8 & .32 & $(.08 / .56)=.737$ \\
\hline & Marginal & $\mathrm{P}\left(\mathrm{y}_{1}\right)$ & .44 & \\
\hline
\end{tabular}

In the above figure, the joint probabilities are obtained by using Equation 1. Then, the marginal probabilities and the conditional probabilities are obtained by using Equations 2 and 3, respectively.

The optimal decision policy is obtained in Figure 2. The expected values for the decisions are obtained by the averaging out procedure (taking the average of the payoffs with the probabilities being used as weights). The optimal decisions are obtained by maximizing the expected payoffs (i.e., the folding back procedure).

Figure 2: The Traditional Decision Tree Solution

\begin{tabular}{l} 
If $\mathbf{y}_{\mathbf{1}}$ : \\
\hline Actions \\
\begin{tabular}{|cccc|}
\hline & States of Nature & & $\mathbf{E V}\left(\mathbf{d}_{\mathbf{j}}\right)$ \\
\hline & $\mathbf{s}_{\mathbf{1}}$ & $\mathbf{s}_{\mathbf{2}}$ & \\
\hline $\mathbf{d}_{\mathbf{1}}$ & $\mathrm{P}\left(\mathrm{s}_{\mathbf{1}} / \mathrm{y}_{\mathbf{1}}\right)=.857$ & $\mathrm{P}\left(\mathrm{s}_{2} / \mathbf{y}_{1}\right)=.143$ & $3,140 *$ \\
$\mathbf{d}_{\mathbf{2}}$ & 7,000 & $-20,000$ & -428 \\
\hline
\end{tabular} \\
\hline
\end{tabular}

\begin{tabular}{l} 
If $\mathbf{y}_{\mathbf{2}}$ : \\
\hline Actions \\
\begin{tabular}{|cccc|}
\hline & States of Nature & $\mathbf{E V}\left(\mathbf{d}_{\mathbf{j}}\right)$ \\
\hline & $\mathbf{s}_{\mathbf{1}}$ & $\mathbf{s}_{\mathbf{2}}$ & \\
\hline $\mathbf{d}_{\mathbf{1}}$ & $\mathrm{P}\left(\mathrm{s}_{1} / \mathbf{y}_{1}\right)=.273$ & $\mathrm{P}\left(\mathrm{s}_{2} / \mathbf{y}_{1}\right)=.727$ & $-12,360$ \\
$\mathbf{d}_{\mathbf{2}}$ & 7,000 & $-20,000$ & $1,910^{*}$ \\
\hline
\end{tabular}
\end{tabular}


As shown in Figure 2, the optimal decision procedure is to select $\mathrm{d}_{1}$ (with $\operatorname{EV}\left(\mathrm{d}_{1}\right)=3,140$ ), if $\mathrm{y}_{1}$ and select $\mathrm{d}_{2}$ (with $\mathrm{EV}\left(\mathrm{d}_{2}\right)=1,910$ ), if $\mathrm{y}_{2}$ (i.e., to accept the process as safe only if the test indicates a SAFE PROCESS). The expected value of this decision procedure is calculated by:

$\mathrm{EV}=(.56) * 3,140+(.44) * 1,910=2,600$.

This EV is reduced by the cost of the test of 500 to obtain 2,100 representing the value of the test.

The solution obtained by the traditional decision trees analysis (Figures 1 and 2) requires a total of 27 operations to obtain the optimal decision policy and its expected value. As shown in Figure 1, 10 of these 27 operations are to reprocess probabilities and obtain the revised probability distributions (4 operations to obtain the joint probabilities, 2 operations to obtain the marginal probabilities, and 4 operations to obtain the conditional probabilities). The remaining 17 operations (see Figure 2) are to execute the traditional rollback method and obtain the expected value of the optimal decision procedure.

Using the modified decision tree analysis, the optimal decision procedure and its expected value for the testing procedure are obtained as in Figure 3. As in the case of the traditional decision trees, the optimal decision procedure is to accept the process as safe only if the test indicates a SAFE PROCESS. The EV of the decision procedure is calculated by:

$\mathrm{EV}=1,760+840=2,600$.

This EV is reduced by the cost of the test of 500 to obtain 2,100 representing the value of the test.

Figure 3: The Modified Decision Tree Solution

\begin{tabular}{|c|c|c|c|}
\hline \multirow{2}{*}{$\begin{array}{l}\text { If } y_{1}: \\
\text { Actions }\end{array}$} & \multicolumn{2}{|c|}{ States of Nature } & \multirow{2}{*}{$\mathbf{E V}\left(\mathbf{d}_{\mathrm{j}}\right)$} \\
\hline & $\begin{array}{c}\mathbf{s}_{\mathbf{1}} \\
\mathrm{P}\left(\mathrm{s}_{1}\right)=.6 \\
\mathrm{P}\left(\mathrm{y}_{1} / \mathrm{s}_{1}\right)=.8 \\
\mathrm{P}\left(\mathrm{y}_{1}, \mathrm{~s}_{1}\right)=.48\end{array}$ & $\begin{array}{c}\mathbf{s}_{\mathbf{2}} \\
\mathrm{P}\left(\mathrm{s}_{2}\right)=.4 \\
\mathrm{P}\left(\mathrm{y}_{1} \mathrm{~s}_{2}\right)=.2 \\
\mathrm{P}\left(\mathrm{y}_{1}, \mathrm{~s}_{1}\right)=.08\end{array}$ & \\
\hline $\begin{array}{l}d_{1} \\
d_{2}\end{array}$ & $\begin{array}{l}7,000 \\
1,000\end{array}$ & $\begin{array}{r}-20,000 \\
3,000\end{array}$ & $\begin{aligned} 3360-1600 & =1760 \\
-480+240 & =-240\end{aligned}$ \\
\hline
\end{tabular}

\begin{tabular}{|c|c|c|c|}
\hline Actions & & & $\operatorname{EV}\left(\mathbf{d}_{\mathrm{i}}\right)$ \\
\hline & $\begin{array}{c}\mathbf{s}_{\mathbf{1}} \\
\mathrm{P}\left(\mathrm{s}_{1}\right)=.6 \\
\mathrm{P}\left(\mathrm{y}_{1} / \mathrm{s}_{1}\right)=.2 \\
\mathrm{P}\left(\mathrm{y}_{1}, \mathrm{~s}_{1}\right)=.12\end{array}$ & $\begin{array}{c}\mathbf{s}_{\mathbf{2}} \\
\mathrm{P}\left(\mathrm{s}_{2}\right)=.4 \\
\mathrm{P}\left(\mathrm{y}_{1} \mathrm{~s}_{2}\right)=.8 \\
\mathrm{P}\left(\mathrm{y}_{1}, \mathrm{~s}_{1}\right)=.32\end{array}$ & \\
\hline $\begin{array}{l}d_{1} \\
d_{2}\end{array}$ & $\begin{array}{l}7,000 \\
1,000\end{array}$ & $\begin{array}{r}-20,000 \\
3,000\end{array}$ & $\begin{aligned} 840-6400 & =-5560 \\
-120+960 & =840\end{aligned}$ \\
\hline
\end{tabular}

Unlike the traditional analysis, the proposed solution (Figure 3) requires only 19 operations (4 operations to obtain joint probabilities and 15 operations to obtain the expected value of the optimal decision procedure). Therefore, the proposed solution saves $8(27$ - 19) operations of the total operations required by the traditional decision tree analysis. This saving results from eliminating the need to calculate 6 probabilities ( 2 marginal and 4 revised probabilities) and saving 2 operations in calculating the $\mathrm{EV}$ of the optimal procedure. 


\section{The Proposed Analysis Vs. Traditional Analysis}

Mathematically, the number of calculations needed by the traditional method can be calculated as follows. Let $\mathrm{n}=$ number of states, $\mathrm{j}=$ number of signals, and $\mathrm{m}=$ number of decisions. The number of operations needed to obtain the conditional probabilities (denoted A) and the operations needed to obtain the optimal solution (denoted B) are given, respectively, by:

$$
\begin{aligned}
\mathrm{A}(\text { Old }) & =j n+j(n-1)+j n \\
& =3 j n-j \\
\mathrm{~B}(\text { Old }) & =j m(2 n-1)+j(m-1)+(2 j-1) \\
& =2 j m n+j-1
\end{aligned}
$$

where, $\mathrm{jn}=$ operations to obtain joint probabilities, $\mathrm{j}(\mathrm{n}-1)=$ operations to obtain marginal probabilities, $\mathrm{jn}=$ operations to obtain the revised probabilities, $\mathrm{jm}(2 \mathrm{n}-1)=$ operations to calculate $\mathrm{EV}$ for all decisions and all signals, $\mathrm{j}(\mathrm{m}-1)=$ operations to obtain the maximum value for all signals, and $(2 \mathrm{j}-1)=$ operations to obtain the EV of optimal decision procedure. The total number of operations is then given by:

Total $(\mathrm{Old})=3 \mathrm{jn}+2 \mathrm{jmn}-1$

The number of operations needed by the modified analysis can be calculated in a similar fashion. The number of operations needed to process the probabilities (A) and the operations needed to obtain the optimal solution (B) are given, respectively, by:

$$
\begin{aligned}
A(\text { New }) & =j n \\
B(\text { New }) & =j m(2 n-1)+j(m-1)+(j-1) \\
& =2 j m n-1
\end{aligned}
$$

where, $\mathrm{jn}=$ operations to obtain joint probabilities, $\mathrm{jm}(2 \mathrm{n}-1)$ and $\mathrm{j}(\mathrm{m}-1)$ are as defined above, and $(\mathrm{j}-1)=$ operations to calculate the EV of the optimal decision procedure. Thus, the total number of operations needed by the new model is given by:

Total $(\mathrm{New})=\mathrm{jn}+2 \mathrm{jmn}-1$

The proposed approach requires the calculation of only jn joint probabilities and eliminates the need to calculate $\mathrm{j}(\mathrm{n}-1)$ marginal and jn conditional probabilities. This results in

Savings $\quad=j(n-1)+j n$

The model also saves $\mathrm{j}$ operations in calculating the expected value of the optimal decision procedure. Therefore, the total savings of operations is given by:

$$
\begin{aligned}
\text { Total Savings }(\text { New }- \text { Old }) & =(2 \mathrm{jn}-\mathrm{j})+\mathrm{j} \\
& =2 \mathrm{jn} .
\end{aligned}
$$

As an example, consider the above problem where $\mathrm{n}=2$ states, $\mathrm{j}=2$ signals, and $\mathrm{m}=2$ decisions. In this case, the total number of operations required by the traditional analysis, total number of operations required by the proposed analysis, and the total savings are, respectively, 27, 19, and 8 operations. This saving of 8 operations represents $29.6 \%(8 / 27)$ of the total operations needed by the traditional decision tree analysis. 
The total savings can be more substantial for larger problems. For example, let $\mathrm{n}=10$ states, $\mathrm{j}=10$ signals, and $\mathrm{m}=2$ decisions. In this case, the total number of operations required by the traditional analysis, total number of operations required by the proposed analysis, and the total savings are, respectively, 699, 499, and 200 operations. This saving of 200 operations represents $28.6 \%$ (200/699) of the total operations needed by the traditional decision tree analysis.

The above analysis assumes one level decision trees, where one level of signals is assumed. As indicated earlier, decision trees can be multi-level if they involve multiple signals. Multilevel decision trees can be symmetrical or non-symmetrical. In the case where the problem is symmetrical, one can use the coalescence procedure which, as noted earlier, reduces the number of operations needed to obtain the solution without affecting the number of operations needed to process probabilities.

The savings obtained by the proposed model over the traditional decision tree analysis for both nonsymmetrical and symmetrical two-level decision trees will be discussed in another paper. Let $\mathrm{n}=$ number of states, $\mathrm{j}_{1}=$ number of first level signals, $\mathrm{j}_{2}=$ number of second level signals, and $\mathrm{m}=$ number of decisions. Then, as shown in another paper, the proposed solution requires the calculation of only $\mathrm{j}_{1} \mathrm{n}$ joint probabilities and eliminates the need to calculate $j_{1} j_{2} n$ joint probabilities, $j_{2}\left(j_{1}-1\right)+j_{1} j_{2}(n-1)$ marginal probabilities, and $j_{2} j_{1}+j_{1} j_{2} n$ conditional probabilities. This results in

$$
\begin{aligned}
\text { Savings } & =\mathrm{j}_{1} \mathrm{j}_{2} \mathrm{n}+\mathrm{j}_{2}\left(\mathrm{j}_{1}-1\right)+\mathrm{j}_{1} \mathrm{j}_{2}(\mathrm{n}-1)+\mathrm{j}_{2} \mathrm{j}_{1}+\mathrm{j}_{1} \mathrm{j}_{2} \mathrm{n} \\
& =\mathrm{j}_{2} \mathrm{j}_{1}+3 \mathrm{j}_{1} \mathrm{j}_{2} \mathrm{n}-\mathrm{j}_{2}
\end{aligned}
$$

The model also saves $\mathrm{j}_{2}$ operations in calculating the expected value of the optimal decision procedure. Therefore, the total savings of operations is given by:

$$
\begin{aligned}
\text { Total Savings (New - Old }) & =\mathrm{j}_{2} \mathrm{j}_{1}+3 \mathrm{j}_{1} \mathrm{j}_{2} \mathrm{n}-\mathrm{j}_{2}+\mathrm{j}_{2} \\
& =\mathrm{j}_{2} \mathrm{j}_{1}+3 \mathrm{j}_{1} \mathrm{j}_{2} \mathrm{n}
\end{aligned}
$$

As an example, consider a symmetrical decision problem where $\mathrm{n}=10$ states, $\mathrm{j}_{1}=10$ signals, $\mathrm{j}_{2}=10$ signals, and $\mathrm{m}=2$ decisions. Because the example involves a symmetrical decision problem, one can use the coalescence procedure. In this case, the total number of operations required by the traditional decision analysis (with coalescence), total number of operations required by the new model (with coalescence), and the total savings are, respectively, 3979, 879, and 3100 operations. This saving of 3100 operations represents $77.9 \%$ (3100/3979) of the total operations needed by the traditional decision tree analysis.

\section{Comparison With Scenario Trees And Game Trees}

As noted in the introduction, some researchers provided modified decision trees that attempt to preserve the advantages of the traditional decision analysis while improving the efficiency of the solution. For example, using von Neumann and Morgenstern's [1944] extensive form representation of n-person games, Shenoy [1993b] suggested the use of a game tree representation for decision analysis. In another paper, Shenoy [1994b] suggested another decision tree representation (called scenario trees) that used a new pruning method for decision trees.

As described by Shenoy [1994b], scenario trees are different from the traditional decision trees. Rather than calculating probabilities for each edge of a chance node, scenario trees calculate probabilities (called path probabilities) for each path from the root node to a leaf node (i.e., for each scenario). Once the path probabilities are calculated, they are multiplied by the payoffs to obtain weighted payoffs for each path. The weighted payoffs are then used to prune chance and decision nodes and obtain the optimal decision procedure. The weighted payoff obtained after all decision nodes are pruned is the expected (payoff) value of the optimal decision procedure. 
As described by Shenoy [1993b], game trees are similar to the traditional decision trees. However, they differ in their sequencing of chance and decision nodes on the paths from the root node to leaf nodes. The solution of the game tree is a modified version of the traditional rollback method used in the traditional decision trees. In Shenoy [1994b], the new pruning method suggested for scenario trees was used with game trees analysis and resulted in a more efficient solution than the solution obtained by using the modified rollback procedure.

To demonstrate the efficiency of games trees and scenario trees over the traditional trees, Shenoy [1994b] compared the traditional tree analysis (without coalescence) and the traditional tree analysis (with coalescence) to game trees (with the traditional rollback method), game trees (with the new pruning method), and scenario trees. He compared the number of calculations required by the five techniques to obtain the optimal decision procedure for an example involving a medical diagnosis problem. The decision is to treat $\left(\mathrm{d}_{1}\right)$ or not treat $\left(\mathrm{d}_{2}\right)$ patients suspected of suffering from disease $\mathrm{S}$. Patients are assumed to have the disease $\left(\mathrm{s}_{1}\right)$ or not have the disease $\left(\mathrm{s}_{2}\right)$. The disease may result in one of two pathological states $\left(\mathrm{y}_{1}, \mathrm{y}_{2}\right)$ which may cause one of two symptoms $\left(\mathrm{k}_{1}, \mathrm{k}_{2}\right)$.

The number of calculations required by the traditional trees (without coalescence) and traditional trees (with coalescence) were 71 and 59, respectively. As calculated by Shenoy [1994b], the number of calculations required by game trees (with the traditional rollback method), game trees (with the new pruning method), and scenario trees were: 63,49 , and 43 , respectively. Thus, the savings from replacing the traditional trees (with coalescence) by game trees (with the new pruning method) and scenario trees were 10 and 16 . However, game trees (with the traditional rollback method) is less efficient than the traditional trees (with coalescence).

By applying the modified decision tree analysis to the medical diagnosis problem, the total number of operations needed to process probabilities and obtain the optimal decision policy were 43 operations without coalescence and only 31 operations when the coalescence procedure is used because the tree is symmetrical. Thus, the new methods of game and scenario trees are not more efficient than the solution proposed in this paper. The savings of the proposed decision tree analysis over game and scenario trees are discussed in another paper.

More importantly, one should note that scenario trees, unlike the proposed and traditional analysis, do not allow for the use of the coalescence procedure in the case of symmetrical decision trees. Therefore, the proposed and traditional analysis may require less operations and, thus, be more efficient than scenario trees for problems where the coalescence procedure can be used. Consider, for example, a problem where $\mathrm{j}_{1}=\mathrm{j}_{2}=\mathrm{n}=\mathrm{m}=8$. In this case, the total number of operations required by scenario trees and the traditional trees (with coalescence) are 8767 and 3647, respectively. Thus, the traditional trees (with coalescence) requires 5120 operations less than scenario trees. The proposed model (with coalescence) requires only 2047 operations and, thus, it saves 6720 operations over scenario trees. This saving represents $76.7 \%$ (6720/8767) of the total operations required by scenario trees.

\section{Summary And Concluding Remarks}

Decision trees and Bayesian decision analysis are widely used as a basis for quality control decision making. Recently, the traditional decision tree analysis has been criticized for requiring a lot of calculations and, therefore, being inefficient. As a result, some researchers provided new decision trees (game and scenario trees). These decision trees attempt to preserve the advantages of the traditional trees while improving their efficiency.

This paper presents a simplified and efficient decision tree analysis that improves the efficiency of the traditional tree analysis. The paper shows that the proposed tree analysis substantially reduces the number of operations required by the traditional analysis by eliminating the need to calculate the conditional probabilities. The proposed modified analysis is also as efficient and in many cases more efficient than game trees and scenario trees. In fact, these other modified decision trees may not be as efficient as the traditional decision tree analysis because they do not allow for the use of the coalescence procedure when the decision problem is symmetrical.

To address the inefficiency of the traditional decision trees, some researchers have suggested the use of influence diagrams (Olmsted [1983], Shachter [1986, 1988], Smith [1989]) and valuation networks [Shenoy, 1989, 
1992] as alternatives to decision trees. These alternative methods are said to be more efficient than the traditional decision trees in that they reduce the number of calculations needed to solve decision problems. However, as shown in another paper, the traditional tree analysis with the modification proposed in this paper is as efficient as valuation networks and requires less calculations and, therefore, more efficient than influence diagrams.

\section{References}

1. Call, H. and Miller, W. (1990). "A Comparison of Approaches and Implementations for Automating decision analysis," Reliability Engineering and Systems Safety, 30, 115-162.

2. Kirkwood, C. (1993). "An Algebraic Approach to Formulating and Solving Large Models for sequential Decisions Under Uncertainty," Management Science, 39, 7 (July), 900-913.

3. Olmsted, S. (1983). "On Representation and Solving Decision Problems," Ph.D. Dissertation, Department of Engineering-economic Systems, Stanford University, Stanford, CA.

4. Quinlan, J. (1987). "Simplifying Decision Trees," International Journal of Man-Machine Studies, 23, 221234.

5. Raiffa, H. (1968). Decision Analysis: Introductory Lectures on Choices Under Uncertainty (Reading, MA: Addison-Wesley).

6. $\quad$ Shachter, R. (1986). "Evaluating Influence Diagrams," Operations Research, 34, 871-882.

7. $\quad$ Shachter, R. (1988). "Probabilistic Influence Diagrams," Operations Research, 36, 589-604.

8. Shenoy, Prakash (1989). "A Valuation-Based Language for Expert Systems," International Journal of Approximate Reasoning, 3, 383-411.

9. Shenoy, Prakash (1992). "Valuation-Based Systems for Bayesian Decision Analysis," Operations Research, 40, 463-484.

10. Shenoy, Prakash (1993a). "A New Method for Representing and Solving Bayesian Decision Problems," in D. Hand (ed.), Artificial Intelligence Frontiers in Statistics: AI and Statistics III (London: Chapman and Hall), 119-138.

11. Shenoy, Prakash (1993b). "Game Trees for Decision Analysis," Working paper No. 239, School of Business, University of Kansas, Lawrence, Kansas. A Summary of the paper appeared as "Information Sets in Decision Theory," in M. Clarke, R. Kruse and S. Moral (eds.), Symbolic and Quantitative Approaches to Reasoning and Uncertainty, Lecture Notes in Computer Sciences No. 747 (Berlin: Springer-verlag, 1993).

12. Shenoy, Prakash (1994a). "A Comparison of Graphical Techniques for Decision Analysis," European Journal of Operational Research, 78, 1-21.

13. Shenoy, Prakash (1994b). "A New Pruning Method for Solving Decision Trees and Game Trees," Working paper No. 261, School of Business, University of Kansas, Lawrence, Kansas.

14. Smith, J. (1989). "Influence Diagrams for Bayesian Decision Analysis," European Journal of Operational Research, 40, 363-376.

15. von Neumann, J. and Morgenstern, O. (1944). Theory of Games and Economic Behavior (New York, NY: John Wiley \& Sons). 
Notes 